\author{
MITSUBISHI ELECTRIC RESEARCH LABORATORIES \\ http://www.merl.com
}

\title{
Random Steerable Arrays for Synthetic Aperture Imaging
}

\author{
Liu, D.; Boufounos, P.T.
}

TR2013-035 May 2013

\begin{abstract}
In classical spotlight-mode synthetic aperture radar (SAR), a mobile sensor array is steered to always focus on the same area (spot) as it moves, transmitting pulses to illuminate the spot and receiving and processing their reflections. The result is a high resolution image, covering a relatively small area. In this paper, we propose using a randomly steerable sensor array for synthetic aperture imaging, aiming to increase the coverage area without sacrificing the imaging resolution. This is realized by steering the beam of the array such that each transmitted pulse illuminates one of two or more spots, randomly selected with equal probability. Each of those spots has the same size as a single spot in a classical array, effectively doubling (or more) the total area illuminated. Using principles from compressive sensing (CS) we demonstrate that it is possible to reconstruct the images of all illuminated areas by exploiting the structure of the reconstructed images. Our experimental results demonstrate that our random steerable array can double coverage with almost the same imaging resolution.
\end{abstract}

IEEE International Conference on Acoustics, Speech and Signal Processing (ICASSP)

\footnotetext{
This work may not be copied or reproduced in whole or in part for any commercial purpose. Permission to copy in whole or in part without payment of fee is granted for nonprofit educational and research purposes provided that all such whole or partial copies include the following: a notice that such copying is by permission of Mitsubishi Electric Research Laboratories, Inc.; an acknowledgment of the authors and individual contributions to the work; and all applicable portions of the copyright notice. Copying, reproduction, or republishing for any other purpose shall require a license with payment of fee to Mitsubishi Electric Research Laboratories, Inc. All rights reserved.
} 



\title{
RANDOM STEERABLE ARRAYS FOR SYNTHETIC APERTURE IMAGING
}

\author{
Dehong Liu, Petros T. Boufounos \\ Mitsubishi Electric Research Labs, 201 Broadway, Cambridge, MA 02139 \\ $\{$ liudh,petrosb\}@merl.com
}

\begin{abstract}
In classical spotlight-mode synthetic aperture radar (SAR), a mobile sensor array is steered to always focus on the same area (spot) as it moves, transmitting pulses to illuminate the spot and receiving and processing their reflections. The result is a high resolution image, covering a relatively small area. In this paper, we propose using a randomly steerable sensor array for synthetic aperture imaging, aiming to increase the coverage area without sacrificing the imaging resolution. This is realized by steering the beam of the array such that each transmitted pulse illuminates one of two or more spots, randomly selected with equal probability. Each of those spots has the same size as a single spot in a classical array, effectively doubling (or more) the total area illuminated. Using principles from compressive sensing (CS) we demonstrate that it is possible to reconstruct the images of all illuminated areas by exploiting the structure of the reconstructed images. Our experimental results demonstrate that our random steerable array can double coverage with almost the same imaging resolution.
\end{abstract}

Index Terms - synthetic aperture radar, synthetic aperture imaging, random steering, compressive sensing

\section{INTRODUCTION}

\subsection{Background}

Synthetic aperture radar (SAR) exploits the motion of moving sensors to form a large synthetic aperture, thus improving the imaging resolution. In spotlight-mode, the sensors are steered to always illuminate a single area (spot) of interest using pulses transmitted at uniform time intervals. The sensor positions, in which pulses are transmitted and reflections are recorded, synthesize a large virtual aperture producing significantly higher imaging resolution compared to physical aperture arrays or strip-map mode synthetic arrays. However, there is a tradeoff between imaging resolution and coverage. Compared to strip-map mode SAR, classical spotlight mode can only cover a much smaller area because of its high sampling rate requirement and restrictions on its beam geometry [1].

In this paper, we modify the fundamental operation of spotlightmode synthetic aperture sensing, to increase the coverage area (also referred to as scene size) without sacrificing imaging resolution. Our approach consists of two major contributions:

- We modify the steering mode of the array such that at any point the beam is randomly steered to illuminate one of two or more different spots in the scene.

- We exploit the structure of the measured scene in the reconstruction process and recover from the loss of data due to the modification in the measurement process.

Specifically, instead of always steering the sensor to illuminate the same spot as it moves across the virtual aperture, we illuminate multiple spots in the measured scene, each with the same size as a single spot in a classical setting, thus multiplying the total area illuminated. In contrast to our earlier work on strip-map mode SAR [2], this approach does not randomize the pulsing times, only the steering. At each pulsing time, we randomly pick one of the candidate spots to illuminate with equal probability. The randomization is necessary to ensure the measurements capture enough information for high resolution image reconstruction. Conceptually this is like missing illumination data from one spot so we can illuminate others. A benefit of uniform pulsing is that the modifications necessary to implement our approach in classical synthetic aperture system are minimal.

When reconstructing each of the spots, we can consider the steerable sensor as operating exactly the same as in classical spotlight-mode, except that only a portion of the data, randomly selected, are recorded for imaging and the remaining is missing. Based on recently developed compressive sensing (CS) techniques [3], we develop a reconstruction algorithm and are able to reconstruct the images of two spots, despite the missing data, by exploiting the sparsity of the underlying reflectivity images.

In this paper, we focus on the CS-based image reconstruction algorithm, assuming the random steering is realized by controlling the beam-pattern electronically or by steering the sensors mechanically [4]. To validate our approach, we provide experimental results using simulated acquisition with both classical spotlight-mode and our proposed randomly-steered system. Although we sometimes describe our work using a two-spot example, extension to multiple spots is straightforward.

\subsection{Relation to prior work}

In recent years, the development of compressive sensing (CS) has had great impact in sensing applications, including radar imaging [5-9]. CS fundamentally revisits signal acquisition and allows robust reconstruction of signals using a significantly smaller number of measurements compared to their Nyquist rate. This sampling rate reduction is achieved by using randomized measurements, improved signal models and non-linear reconstruction algorithms.

Although CS has enabled significant improvements on radar and radar imaging systems $2,10,13$, a number of challenges still exist in applying CS to radar imaging, such as developing appropriate sparsity models of radar images and managing computational complexity [5, 6,14$]$.

Our proposed approach is related to existing classical and CSbased radar arrays, appropriately modifying each to improve imaging capabilities. Our non-uniformly steered array is based on classical steerable SAR, but with a very different steering pattern. Our proposed architecture is straightforward to implement, even in existing synthetic aperture systems.

Our approach is also reminiscent of our earlier work on stripmap- 


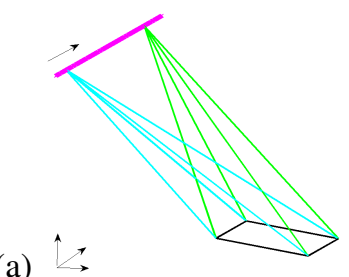

(a)

Fig. 1. Spotlight steering, illustrating the path of the mobile platform and the illumination of the scene along the path at different locations. (a) A classical array in spotlight mode always illuminated the same spot. (b) The proposed randomly-steered spotlight mode randomly alternates illuminating one of two or more spots.

mode arrays [2], but it has significant differences. In that work, the pulse timing of a non-steerable stripmap-mode SAR is randomized to enable a larger range acquisition with no compromise in the resolution. This causes interference in the received data from overlapped reflections and missing data within a single reflection, which should be appropriately handled in the reconstruction. Our current approach does not exhibit such issues; for each spot area, the transmitting and receiving process is identical to classical spotlight array, except that only a portion of the range line data are collect for each spot.

Furthermore, our reconstruction algorithm incorporates sparse modeling and least square estimation by decomposing the underlying image into sparse part and dense part. Our proposed algorithm outperforms algorithms using only sparsity regularization. While we demonstrate in a two-spot example, the results can be extended to three or more spots with simple modifications.

The paper is organized as follows. In the next section we provide a brief overview on synthetic aperture imaging data acquisition, with emphasis on spotlight mode. Section 3 describes how a randomly steerable array can be utilized to increase array coverage. In Section 4 we briefly examine CS-inspired image formation algorithms to reconstruct from the acquired reflections. Experimental results that confirm and validate our approach are provided in Section 5 and conclusions in Section 6

\section{BACKGROUND OF SPOTLIGHT ARRAY}

We consider general spotlight SAR imaging using a linear monostatic array, operating as depicted in Fig. 1. a). To image an area or scene, a mobile platform moves along the path depicted, transmits pulse at a uniform pulsing rate and receives and records the reflection of those pulses from the imaged area. In spotlight mode, the transmitted pulse beam is steered such that its main lobe aims at the center of the area of interest. Each reflection from the area of interest is effectively a convolution of the transmitted pulse with the reflectivity of the area illuminated by the pulse. Thus, the data acquisition process can be modeled as a linear operation:

$$
\mathbf{y}=\mathbf{\Phi} \mathbf{x}+\mathbf{n},
$$

where $\mathbf{y}$ denotes the received radar echoes, $\mathbf{x}$ denotes the reflectivity of the imaged area, $\boldsymbol{\Phi}$ models the array acquisition function of the array parameters, and $\mathbf{n}$ is measurement noise.

The goal of the image formation process is to determine the signal of interest $\mathbf{x}$ from the array echoes $\mathbf{y}$ given the acquisition function $\boldsymbol{\Phi}$. In other words, it solves an inverse problem. If the acquisition function $\boldsymbol{\Phi}$ is invertible, an obvious choice is to use the inverse or the pseudoinverse of $\boldsymbol{\Phi}$ to determine $\mathbf{x}$ :

$$
\hat{\mathbf{x}}=\boldsymbol{\Phi}^{\dagger} \mathbf{y}
$$

In practical synthetic aperture array systems, $\boldsymbol{\Phi}$ is generally difficult to model accurately and the inversion can be computationally expensive. Typically, array image formation is achieved using one of the well-established algorithms, such as the chirp-scaling algorithm and the wave-number algorithm, which approximate the inversion. Considering the large squint angle in spotlight arrays, we employ the wave-number algorithm to approximate the inversion problem [1, 15].

\section{RANDOMLY STEERABLE ARRAY}

Our randomly steerable array operates with the same principles as the classical spotlight-mode SAR, uniformly transmitting pulses and receiving echoes, except that it incorporates more flexibility in steering. The operation is illustrated in Fig. 1 b). Instead of steering the mobile sensor to always illuminate the same area as the sensor moves, we illuminate two or more spots, both having the same size as the size of a single spot in a classical system. The example demonstrates how illuminating two adjacent spots results in illuminating an area of doubled size. At different transmitting/receiving locations, we randomly pick one of the candidate spots to illuminate with equal probability. In the example, each of the two spots has the same size as classical array coverage, but is illuminated by only half the pulses, leaving the other half for the other spot.

As we discuss in Section 1 for each of the areas, the randomly steerable system can modeled exactly the same as classical spotlightmode SAR, except that part of the echoes are missing. Thus, similar to the classical spotlight sensing, we can describe the data acquisition process as a linear operation with missing data. If the array was always steered towards area $i$, it would acquire $\mathbf{x}_{i}$ through linear acquisition function $\boldsymbol{\Phi}_{i}$. However, it misses some of the acquired data, according to how the array is steered. We denote this selection process using $\mathbf{E}_{i}$, an operator selecting only the data actually acquired (measured). Further, we use $\overline{\mathbf{E}}_{i}$ to denote the complementary operator, i.e., the one selecting only the data that are not acquired (i.e., missing or unmeasured). Using $\mathbf{y}_{m i}$ and $\mathbf{y}_{u i}$ to denote the measured and unmeasured data, respectively, we have

$$
\begin{aligned}
\mathbf{y}_{m i} & =\mathbf{E} \boldsymbol{\Phi}_{i} \mathbf{x}_{i}+\mathbf{n}, \\
\mathbf{y}_{u i} & =\overline{\mathbf{E}} \boldsymbol{\Phi}_{i} \mathbf{x}_{i} .
\end{aligned}
$$

Note that the $\mathbf{E}_{i}$ are complementary, i.e., the portion of the data measured from area $i$ cannot also be measured from area $j \neq i$, and the sum of $\mathbf{E}_{i}$ is equal to the identity.

Our goal is to image all spots, even with missing data, without compromising the imaging resolution. This is possible using CS-based methods that exploit the structure of the measured scene-typically in the form of sparsity under some appropriate basis transformation - and randomness in the acquisition process to enable accurate reconstruction. The steering randomization ensures that the linear measurements are less correlated and fully capture the information in the scene. Thus the measurements can be inverted using a non-linear reconstruction process which exploits a signal model to recover the acquired signal.

\section{CS-BASED IMAGE RECONSTRUCTION}

In this section, we focus on an algorithm reconstructing each area separately using only the part of the data measured from this area. 
For notation convenience we drop the subscript $i$ from the remaining development and we operate the algorithm in the same manner for each area $i$.

Using the notation above without the noise component, the full data, including measured and unmeasured, can be represented as

$$
\mathbf{y}=\left[\begin{array}{l}
\mathbf{y}_{m} \\
\mathbf{y}_{u}
\end{array}\right]=\left[\begin{array}{l}
\mathbf{E} \\
\mathbf{E}
\end{array}\right] \mathbf{\Phi x} .
$$

In classical CS, $\mathbf{x}$ is generally treated as a sparse signal, a model generally not accurate for radar imaging. While strong components in some domain might exist in radar imaging, the residual always seems large and difficult to take into account. Instead, we propose a CS-based algorithm that, instead of simply treating $\mathbf{x}$ as a sparse signal, we decompose $\mathbf{x}$ into sparse part $\mathbf{x}_{s}$ and dense residual $\mathbf{x}_{r}$ :

$$
\mathbf{x}=\mathbf{x}_{s}+\mathbf{x}_{r} .
$$

Substituting (6) into (5), the noisy measured data is

$$
\mathbf{y}_{m}=\mathbf{E} \boldsymbol{\Phi} \mathbf{x}_{s}+\mathbf{E} \boldsymbol{\Phi} \mathbf{x}_{r}+\mathbf{n}
$$

Treating $\mathbf{E} \boldsymbol{\Phi} \mathbf{x}_{r}$ as additional noise, the estimate of the sparse component $\mathbf{x}_{s}$ is

$$
\widehat{\mathbf{x}}_{s}=\arg \min _{\mathbf{x}}\|\mathbf{y} m-\mathbf{E} \mathbf{\Phi} \mathbf{x}\|_{2}^{2} \text { s.t. }\|\mathbf{x}\|_{0}<N .
$$

Given the estimate $\widehat{\mathbf{x}}_{s}$, we can estimate its contribution to the measured data $\mathbf{E} \boldsymbol{\Phi} \widehat{\mathbf{x}}_{s}$. Assuming the residual data $\mathbf{y}_{m}-\mathbf{E} \boldsymbol{\Phi} \widehat{\mathbf{x}}_{s}$ is due to $\mathbf{x}_{r}$, we can obtain its least squares estimate using

$$
\widehat{\mathbf{x}}_{r}=(\mathbf{E} \boldsymbol{\Phi})^{\dagger}\left(\mathbf{y} m-\mathbf{E} \boldsymbol{\Phi} \widehat{\mathbf{x}}_{s}\right) .
$$

We then have the final image by combining (8) and 9

$$
\widehat{\mathbf{x}}=\widehat{\mathbf{x}}_{s}+\widehat{\mathbf{x}}_{r}=\widehat{\mathbf{x}}_{s}+(\mathbf{E \Phi})^{\dagger}\left(\mathbf{y} m-\mathbf{E} \boldsymbol{\Phi} \widehat{\mathbf{x}}_{s}\right) \text {. }
$$

Note that the final image is not sparse, but a combination of a sparse component, estimated using sparsity regularization, and a dense component estimated using least-squares regularization. In our experiments on synthetic aperture images, this reconstruction approach outperformed reconstruction approaches using only sparsity regularization.

Letting $\widehat{\mathbf{y}}_{s}$ denote the full data corresponding to $\widehat{\mathbf{x}}_{s}$, i.e.,

$$
\widehat{\mathbf{x}}_{s}=\boldsymbol{\Phi}^{\dagger} \widehat{\mathbf{y}}_{s}
$$

we rewrite 10 as follows

$$
\widehat{\mathbf{x}}=\boldsymbol{\Phi}^{\dagger} \widehat{\mathbf{y}}_{s}+(\mathbf{E} \boldsymbol{\Phi})^{\dagger}\left(\mathbf{y}_{m}-\mathbf{E} \boldsymbol{\Phi} \widehat{\mathbf{x}}_{s}\right)
$$

This solution is equivalent to filling in the missing data using the reconstructed by enforcing the sparsity model only, and performing classical least-squares imaging on the completed data. Note that $\mathbf{E}$ is a selection operator, i.e., $\mathbf{E}^{\dagger}=\mathbf{E}^{T}$, i.e. its pseudoinverse just fills zero for the missing data.

Based on this idea, we formulate our algorithm as shown in Fig. 22 In each iteration the algorithm uses the residual $\mathbf{y}_{m r}^{(k-1)}$ to compute an estimate of the so-far unexplained signal $\tilde{\mathbf{x}}^{(k)}$. To obtain the strongest reflectors, a threshold $\tau^{(k)}$ is computed as a fraction of the largest in magnitude signal component. The estimate of the strongest reflectors $\mathbf{d}^{(k)}$ is computed by imposing a hard threshold $\mathcal{H}_{\tau^{(k)}}(\cdot)$ on $\tilde{\mathbf{x}}^{(k)}$, i.e., by setting all components less than $\tau^{(k)}$ in magnitude to zero. This estimate is scaled using $\beta^{(k)}$ such that it explains most of the residual energy in $\mathbf{y}_{m r}^{(k-1)}$. It is then added to
1. Initialize $0<\alpha<1, \widehat{\mathbf{x}}_{s}^{(0)}=\mathbf{0}, \mathbf{y}_{m r}^{(0)}=\mathbf{y}_{m}$,

2. FOR $k=1: K$

$$
\begin{aligned}
\tilde{\mathbf{x}}^{(k)} & =\boldsymbol{\Phi}^{\dagger} \mathbf{E}^{\dagger} \mathbf{y}_{m r}^{(k-1)} \\
\tau^{(k)} & =\max \left(\left|\tilde{\mathbf{x}}^{(k)}\right|\right) \cdot \alpha \\
\mathbf{d}^{(k)} & =\mathcal{H}_{\tau^{(k)}}\left(\tilde{\mathbf{x}}^{(k)}\right) \\
\tilde{\mathbf{y}}^{(k)} & =\mathbf{E} \boldsymbol{\Phi} \mathbf{d}^{(k)} \\
\beta^{(k)} & =\frac{<\tilde{\mathbf{y}}^{(k)}, \mathbf{y}_{m r}^{(k-1)}>}{<\tilde{\mathbf{y}}^{(k)}, \tilde{\mathbf{y}}^{(k)}>} \\
\mathbf{y}_{m r}^{(k)} & =\mathbf{y}_{m r}^{(k-1)}-\beta^{(k)} \tilde{\mathbf{y}}^{(k)} \\
\widehat{\mathbf{x}}_{s}^{(k)} & =\widehat{\mathbf{x}}_{s}^{(k-1)}+\beta^{(k)} \mathbf{d}^{(k)}
\end{aligned}
$$

\section{END}

\section{Output}

$$
\begin{aligned}
\text { Echoes: } \widehat{\mathbf{y}} & =\mathbf{E}^{\dagger} \mathbf{y}_{m}+\overline{\mathbf{E}}^{\dagger} \boldsymbol{\Phi} \widehat{\mathbf{x}}_{s}^{(K)} \\
\text { Image: } \widehat{\mathbf{x}} & =\boldsymbol{\Phi}^{\dagger} \widehat{\mathbf{y}}
\end{aligned}
$$

Fig. 2. Reconstruction algorithm

the overall signal estimate from the previous iteration $\widehat{\mathbf{x}}_{s}^{(k-1)}$ to produce the current signal estimate $\widehat{\mathbf{x}}_{s}^{(k)}$ and subtracted from the residual $\mathbf{y}_{m r}^{(k-1)}$ to produce the updated residual $\mathbf{y}_{m r}^{(k)}$. As the last step, after the iterations are concluded, the algorithm uses the estimated signal $\widehat{\mathbf{x}}_{s}^{(K)}$ from the $K^{t h}$ iteration to estimate the full data $\widehat{\mathbf{y}}$ from which it estimates the final image $\widehat{\mathbf{x}}$ using a classical imaging algorithm.

Summing up, our algorithm attempts to express the sparse component as a linear combination of spatially sparser components of decreasing intensity in the measurements, corresponding to the most intense reflectors. To efficiently compute the imaging process $\boldsymbol{\Phi}^{\dagger}$, we implement the wavenumber algorithm [1]. The forward process $\Phi$ can also be computed with the same efficiency with minor modifications [16]. To achieve relatively good imaging performance and fast execution we found that $\alpha>0.5$ is a good parameter choice.

This algorithm is inspired by STOMP [17], but differs in estimating the sparse signal in each iteration. In our algorithm, we do not compute the psudo-inverse of a subset of $\boldsymbol{\Phi}$; this is computationally expensive since $\boldsymbol{\Phi}$ is typically very large in imaging applications. Instead, similarly to the matching pursuit (MP) [18], we use the signal value after thresholding, scaled by $\beta^{(k)}$, as an estimate of the sparse signal. We found that this heuristic choice provides a good trade-off between speed and accuracy compared to classical CS algorithms.

\section{EXPERIMENTS}

To test our approach, we simulated echoes using a random steerable spotlight synthetic aperture system. We tested our approach on an idealized area with isolated targets as well as a more realistic scene with complex-valued reflectivity. We considered the case of steering between two areas, effectively doubling the area of the acquired 
(a)

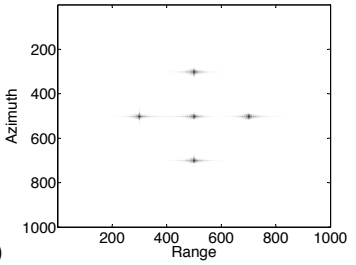

(c)

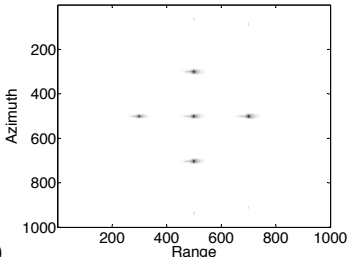

(d)

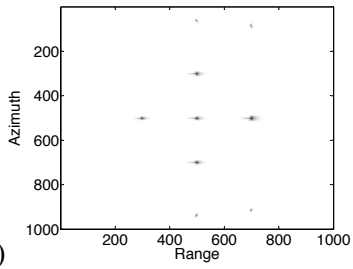

(b)

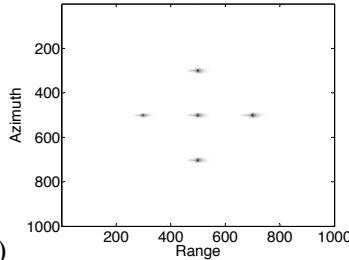

Fig. 3. (a) Classical spotlight imaging result, (b) classical spotlight array imaging with uniform half downsampled data, (c) iterative CS imaging on uniform half downsampled data, (d) iterative CS imaging on random half data collected by random steerable array.

scene. To demonstrate the necessity of random steering we examined two different steering strategies: alternating between the two spots sequentially for each pulse, and randomly steering to one spot or the other at each pulse. The former is equivalent to uniformly undersampling by 2 the full measurements from each spot while the latter is equivalent to randomly undersampling the same measurements by the same average rate. To demonstrate the necessity of a sparse reconstruction algorithm, we examine how our algorithm performs on the uniformly undersampled data, compared to classical imaging algorithms.

For the first experiment, we consider an area with five isolated scatterers. Due to space limitations, we only show imaging results for one of the two spots. Because of the symmetry of the steering methods, the performance on the other spot is similar. Figure 3 shows the results. In particular, Fig. 3 a) shows the inversion using full data, i.e., assuming the array is always steered to the same spot. Figure 3 b) shows the reconstruction from uniformly undersampled data, using a classical reconstruction method. The spatial aliasing (ghosting) and the resulting ambiguity is prominent in the picture. Figure 3 c) demonstrated the reconstruction using our algorithm. While the ghosting due to spatial aliasing has not been eliminated, it has been significantly attenuated. Finally, Fig. 3.d) shows the reconstruction from a randomly sampled spot using our algorithm. As evident, our approach does not exhibit any ghosting.

For the second set of experiments we used a real scene of complex valued reflectivity, available at [19]. We perform the same experiments as above, shown in Fig. 4 When using our reconstruction algorithm we set $\alpha=0.75$ and $K=200$ such that for each iteration very few strong scatterers are retained. The imaging results for classical spotlight-mode synthetic aperture imaging are shown in Fig. 4 a). If we use half the measurements, uniformly downsampled, to achieve double sized areas, the image exhibits significant ambiguity in the form of ghosting, similar to the previous experiment, shown in Fig. 4(b). Using a randomly steerable spotlight and a wavelet sparsity model [2] we obtain the image in Fig. 4 (c), which is improved but still not satisfactory. Using our proposed CS-based steering and reconstruction, the imaging result is significantly improved, as shown in Fig. 4 (d) and very close the one in Fig. 4 (a) where full data is available.

For the other spot area, we observe similar imaging performance (a)

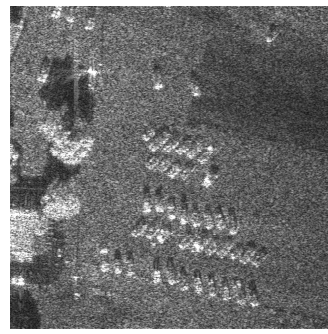

(c)

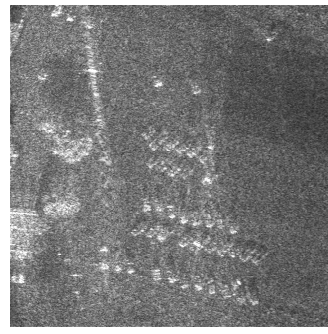

(d)
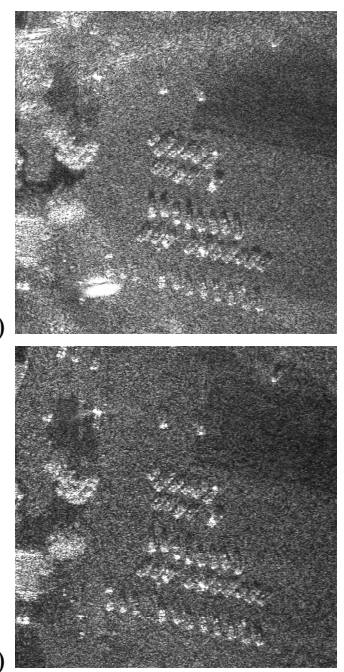

Fig. 4. (a) Classical spotlight imaging result, (b) classical spotlight array imaging with uniform half downsampled data, (c) imaging on random half data using wavelet sparsity model, (d) imaging on random half data using proposed algorithm.

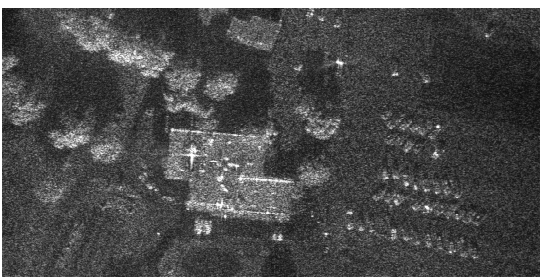

(a)

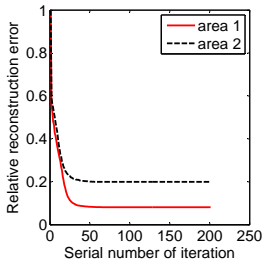

(b)
Fig. 5. (a) Combined view for two spots, (b) relative reconstruction error of two spots.

using the other half of the data. Combining together the imaging results of the two spot areas, we obtain double the coverage as shown in Fig. 5(a), and almost the same resolution with the relative reconstruction error plotted in Fig. 5(b).

\section{CONCLUSION}

This paper describes a randomly steerable synthetic aperture imaging system to increase the spotlight-mode SAR coverage without compromising the imaging resolution. Instead of steering the spotlight to always illuminate only one area, it is steered randomly to illuminate one of many possible. This randomization removes ambiguities that lead to spatial aliasing and ghosting. To form the image from the acquired data we propose an iterative reconstruction algorithm which combines sparsity-promoting and least squares estimation. Our experimental results validate our method and show that it is possible to double the coverage area with minimal resolution penalty.

As regarding to future research work, there are several open questions on the performance of the system, such as the trade-off between the area covered, the sparsity of the scene and the resolution achievable by the system. Several improvements on the reconstruction algorithm are also possible. These and other pertinent questions are reserved in future publications. 


\section{REFERENCES}

[1] I. G. Cumming and F. H. Wong, Digital processing of synthetic aperture radar data: algorithms and implementation, Artech House, 2005.

[2] D. Liu and P. T. Boufounos, "High resolution SAR imaging using random pulse timing," in IEEE International Geoscience and Remote Sensing Symposium (IGARSS), 2011.

[3] E. Candes, J. Romberg, and T. Tao, "Robust uncertainty principles: Exact signal reconstructoin from highly incomplete frequency information," IEEE Transactions on Information Theory, vol. 52(2), Februray 2006.

[4] W. G. Carrara, R. S. Goodman, and R. M. Majewski, Spotlight Synthetic Aperture Radar - Signal Processing Algorithms, Artech House, 1995.

[5] R. Baraniuk and P. Steeghs, "Compressive radar imaging," in IEEE Radar Conference, MA, April 2007.

[6] L. C. Potter, E. Ertin, J. T. Parker, and M. Cetin, "Sparsity and compressed sensing in radar imaging," Processings of the IEEE, vol. 98, pp. 1006-1020, June 2010.

[7] Y. S. Yoon and M. G. Amin, "Compressed sensing techniques for high-resolution radar imaging," in Proc. SPIE 6968, Signal Processing, Sensor Fusion, and Target Recognition XVII, April 2008.

[8] L. Anitori, M. Otten, and P. Hoogeboom, "Compressive sensing for high resolution radar imaging," in Asia-Pacific Microwave Conference Proceedings(APMC), Dec 2010, pp. 1809-1812.

[9] H. Bu, X. Bai, and R. Tao, "A novel algorithm for synthetic aperture radar imaging based on compressed sensing," in IEEE 10th international conference on Signal Processing (ICSP), June 2010, pp. 2210-2213.

[10] V. M. Patel, G. R. Easley, D. M. Healy Jr., and R. Chellappa, "Compressed synthetic aperture radar," IEEE Journal of selected topics in signal processing, vol. 4, pp. 244-254, April 2010.

[11] M. A. Herman and T. Strohmer, "High-resolution radar via compressed sensing," IEEE Trans. Signal Process., vol. 57, June 2009.

[12] M. Tello Alonso, P. Lopez-Dekker, and J. J. Mallorqui, "A novel strategy for radar imaging based on compressive sensing," IEEE Trans. Geoscience and Remote Sensing, vol. 48(12), pp. 4285-4295, Dec 2010.

[13] S. Shah, Y. Yao, and A. Petropulu, "Step-frequency radar with compressive sampling(sfr-cs)," in IEEE International conference on Acoustics Speech and Signal Processing(ICASSP), March 2010.

[14] J. H. G. Ender, "On compressive sensing applied to radar," Signal Processing, vol. 90(5), pp. 1402-1414, May 2010.

[15] D. W. Hawkins, "Synthetic aperture imaging algorithms: with application to wide bandwidth sonar," Ph.D Thesis, October 1996.

[16] D. Wei and P. T. Boufounos, "Saturation-robust SAR image formation," in IEEE International conference on Acoustics Speech and Signal Processing(ICASSP), 2011.
[17] D.L. Donoho, Y. Tsaig, I. Drori, and J.-L. Starck, "Sparse solution of underdetermined systems of linear equations by stagewide orthogonal matching pursuit," IEEE Trans. Information Theory, Februray 2012.

[18] S. Mallat and Z. Zhang, "Matching pursuit with timefrequency dictionaries," IEEE Trans. Signal Process., vol. 41(12), Dec. 1993.

[19] Sandia National Laboratories, "Sandia SAR data," available at http://www.sandia.gov/radar/sar-data.html. 